\title{
Off-Grid Radar Coincidence Imaging Based on Variational Sparse Bayesian Learning
}

\author{
Xiaoli Zhou, Hongqiang Wang, Yongqiang Cheng, and Yuliang Qin \\ School of Electronic Science and Engineering, National University of Defense Technology, Changsha 410073, China \\ Correspondence should be addressed to Hongqiang Wang; oliverwhq@vip.tom.com
}

Received 24 October 2015; Revised 15 March 2016; Accepted 31 March 2016

Academic Editor: Erik Cuevas

Copyright ( 2016 Xiaoli Zhou et al. This is an open access article distributed under the Creative Commons Attribution License, which permits unrestricted use, distribution, and reproduction in any medium, provided the original work is properly cited.

\begin{abstract}
Radar coincidence imaging (RCI) is a high-resolution staring imaging technique motivated by classical optical coincidence imaging. In RCI, sparse reconstruction methods are commonly used to achieve better imaging result, while the performance guarantee is based on the general assumption that the scatterers are located at the prediscretized grid-cell centers. However, the widely existing off-grid problem degrades the RCI performance considerably. In this paper, an algorithm based on variational sparse Bayesian learning (VSBL) is developed to solve the off-grid RCI. Applying Taylor expansion, the unknown true dictionary is approximated accurately to a linear model. Then target reconstruction is reformulated as a joint sparse recovery problem that recovers three groups of sparse coefficients over three known dictionaries with the constraint of the common support shared by the groups. VSBL is then applied to solve the problem by assigning appropriate priors to the three groups of coefficients. Results of numerical experiments demonstrate that the algorithm can achieve outstanding reconstruction performance and yield superior performance both in suppressing noise and in adapting to off-grid error.
\end{abstract}

\section{Introduction}

Motivated by classical coincidence imaging, radar coincidence imaging (RCI) is a novel high-resolution imaging technique which has been realized in optical systems [1-3]. The RCI can realize staring imaging without the requirement of the target relative motion and operate under the nonideal observing geometry of forward-looking/staring and shorten the imaging time to even a single pulse width, with significant potentials for resolution enhancement, interference, and jamming suppression. The basic principle of RCI is to excite timespace independent signals in the imaging area. Thus the spatial variety of wavefront is increased, and the scatterers within a beam then reflect independent signals associated with their respective positions, so the super-resolution within a beam emerges.

To achieve better imaging performance, sparse reconstruction is commonly used, and the continuous space needs to be discretized to a fine grid and target-scattering centers are assumed to be exactly located at the center of these prediscretized grids [3]. Based on the model, the independent detecting signals at different grid-cell centers can be formed as the atoms of sparse representation dictionary. Meanwhile, the scatterers of target are often distributed sparsely in most radar imaging applications; thus sparse reconstruction and compressive sensing (CS) $[4,5]$ are suitable for RCI by exploiting the sparsity of target. In sparse reconstruction theory, signal reconstruction depends on presetting an appropriate sparsifying dictionary which is supported on the prediscretized grids and defines the signal sparsity. However, as the scatterers distributed in a continuous scene are generally located off the grid-cell centers, the off-grid problem emerges no matter how fine the grid is [6], and the performance of RCI would degrade significantly.

The effect of general dictionary mismatch, which is the direct consequence of off-grid effect, is analyzed in [7-10]. This mismatch causes the performance of conventional sparse reconstruction methods to degrade considerably $[3,6,7,11-$ 14]. An intuitive way to sidestep off-grid effect is to work directly on the continuous parameter space, that is, atomic norm minimization approach [15], continuous basis pursuit (CBP) [16]. Considering the off-grid problem, several 
algorithms have been proposed to alleviate the effect. One simple approach is to use refinement strategy and decrease the grid size [17]. Nevertheless, a finer grid may enhance the coherence between the columns of dictionary and increase the computational complexity and instability of reconstruction [14]. Modeling the off-grid problem as a multiplicative perturbation, the sparse total least squares (S-TLS) [18] and joint correlation-parameterization (JCP) [3] algorithms are proposed. To explore the structure of dictionary mismatch, the support-constrained orthogonal matching pursuit (SCOMP) [13] and joint sparse signal recovery methods [19] are proposed based on the first-order Taylor expansion to utilize the support constraint. Lately, from the sparse Bayesian learning (SBL) perspective, several approaches are proposed, such as off-grid sparse Bayesian inference (OGSBI) [20], sparse adaptive calibration recovery via iterative maximum a posteriori (SACR-iMAP) [6], and block SBL (BSBL) [21]. The merit of SBL is its flexibility in modeling sparse signals that can not only promote the sparsity but also exploit the possible structure of the signal to be recovered [20].

Although the performance of OGSBI and SACR-iMAP is outstanding, the prior distribution of off-grid error (OGE) is not fully utilized; the point estimate rather than distribution of OGE is obtained. Adopting the ideas of variational expectation-maximization (EM) and variational Bayesian inference (VBI) described in [7, 22, 23], the off-grid RCI is investigated in the framework of variational sparse Bayesian learning (VSBL). As for SBL, inference in SBL model is not tractable in closed form; thus approximations are needed, such as maximizing the marginal likelihood (MML), EMSBL, and VBI [24]. Compared to other approximations, VBI has several advantages when applied to SBL. First, the distributions rather than point estimates of the unobserved variables can be obtained. Second, VSBL allows obtaining analytical approximations to the posterior distributions of interest even when their exact expressions are intractable. Finally, the VSBL methodology allows using different prior distributions and is a deterministic approximate inference framework that can be applied to many models.

In this paper, based on the first-order Taylor expansion, the off-grid RCI model in range-azimuth space is reformed to be sparsely approximated using atoms from three different dictionaries, and meanwhile the three groups of approximation coefficients share the same support. By assigning appropriate priors to the approximation coefficients, such a model can be conveniently manipulated to recover sparse coefficients under the VSBL framework. Compared with the aforementioned algorithm discussed above, the proposed algorithm imposes a group structure on the coefficient vector and explores the group-sparse structure using VSBL. Numerical experiments show that the algorithm realizes the target reconstruction robustly and achieves both high-resolution and outstanding imaging quality in the presence of off-grid scatterers and is also simple to implement.

The rest of the paper is organized as follows. In Section 2, the off-grid RCI model in the range-azimuth space is presented. Section 3 presents the off-grid variational sparse Bayesian learning (OG-VSBL) algorithm in detail. In Section 4 , the performance of the presented algorithm is verified by numerical examples and compared with some existing sparse reconstruction methods. Finally, some conclusions and future work are discussed in Section 5.

Notations used in this paper are as follows. We use boldface lowercase letters for vectors and boldface uppercase letters for matrices. $(\cdot)^{T},(\cdot)^{H},(\cdot)^{-1}$, and $(\cdot)^{\dagger}$ denote the transpose, conjugate transpose, inverse, and pseudoinverse of a vector or matrix, respectively. $\odot$ and $\operatorname{vec}(\cdot)$ are the Hadamard product and vectorization operation, separately. $\|\cdot\|_{1}$ and $\|\cdot\|_{2}$ denote the $\ell_{1}$ norm and $\ell_{2}$ norm of a vector. $\operatorname{det}(\cdot)$ is the determinant of a matrix; $\operatorname{diag}(\cdot)$ is a matrix with the elements of a vector on the main diagonal. Finally, $\langle\cdot\rangle$ denotes the expectation of a variable.

\section{Problem Formulation}

2.1. Signal Model. As a novel imaging technique, RCI has shown its potentials in high-resolution, staring, and instantaneous imaging [1]. In RCI, the target is illuminated by timespace independent signals, and then the echo components reflected by scatterers at different positions are mutually independent, which could result in the super-resolution within a beam. The RCI can be realized by a multiple-input multipleoutput (MIMO) radar system to transmit time-independent and group-orthogonal waveforms (e.g., stochastic waveforms) [1]. While compared with conventional MIMO radar which focuses on multiple paths or multiple observation angles, RCI needs the interference of transmitted waveforms to make the wavefront show spatial fluctuations, thus the spatial variety of detecting signals increases. In addition, the components of each path are separated utilizing the waveform orthogonality in conventional MIMO radar, while the components are not separated in the whole RCI procedure.

In this paper, a RCI system with $M_{T}$ transmitters and $M_{R}$ receivers is considered; each transmitter emits an independent stochastic waveform. Without loss of generality, the target is assumed to be composed of several ideal point scattering centers without the spatial property for an enough high carrier frequency, which is widely used in the imaging radar system. Furthermore, the scatterers are widely separated to provide some performance guarantee.

The target scene is considered to be a 2D space using polar coordinate as illustrated in Figure 1. The sparse-based RCI methods discretize the continuous target scene and generate a number of grid-cells. Thus the scene is discretized in rangeazimuth space with $U$ azimuth cells, $V$ range cells, and associated cell size $\Delta_{\theta}, \Delta_{R}$. Hence, the number of grid-cells is $K=U V$. Assume that the scatterers are initially located at the grid-cell centers; thus the $k$ th scatterer is located at the rangeazimuth pair $\mathbf{r}_{k}=\left(\theta_{k}, R_{k}\right)$. As the scatterers possess nonzero complex scattering coefficient $\beta_{k}$ which is proportional to the radar cross section (RCS), the associated $\beta_{k}=0$ means that there is no scatterer at the $k$ th grid-cell center. Denote by $S=$ $\left\{k \in\{1,2, \ldots, K\}: \beta_{k} \neq 0\right\}$ the index set of scatterer locations.

Note that the echo is a linear combination of all the scatterers reflected waveforms from all the transmitters. As it is unknown a priori which $\beta_{k}$ is nonzero, we sum over all $K$ possible locations, although contributions to the echo only 


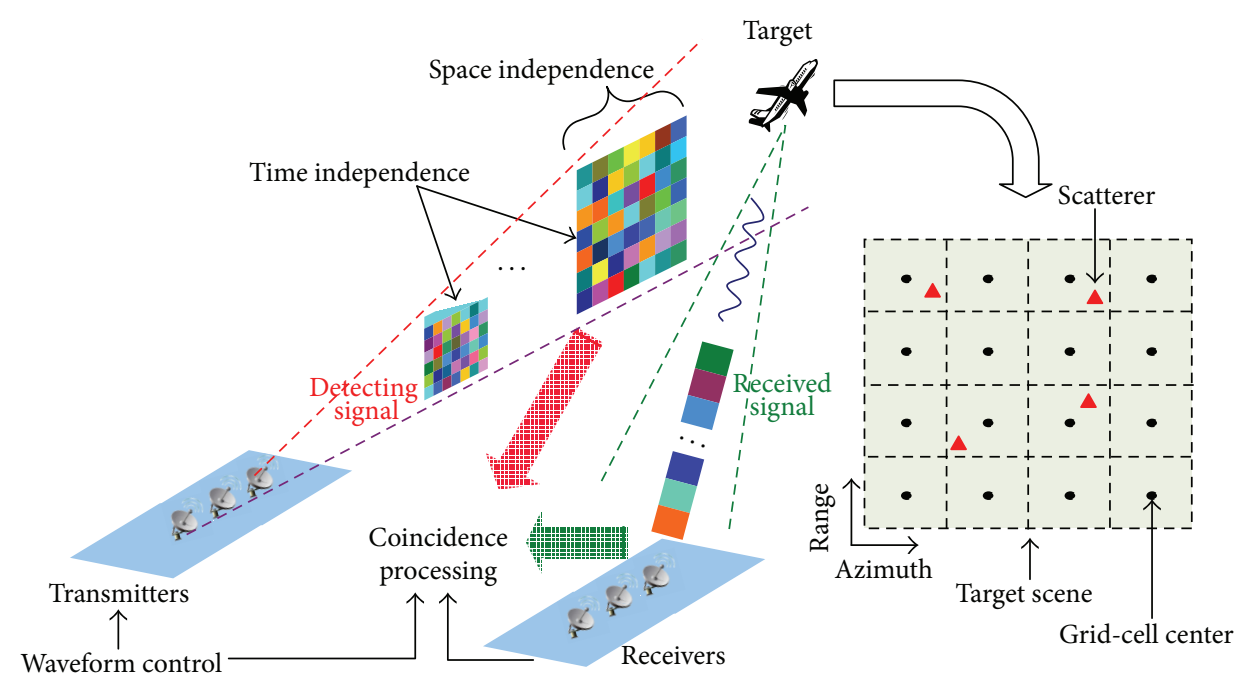

Figure 1: Off-grid RCI geometry.

result from the $P$ scatterers. Then, the echo at the $n$th receiver can be expressed as

$$
y_{n}(t)=\sum_{k \in S} \sum_{m=1}^{M_{T}} \beta_{k} \cdot S t_{m}\left(t-\tau_{m, n}^{k}\right)+w_{n}(t),
$$

where $S t_{m}(t)$ is the signal emitted by the $m$ th transmitter, $w_{n}$ is an independent complex Gaussian random process with zero-mean, and variance $\sigma_{n}^{2}$ denotes the background noise at the $n$th receiver. $\tau_{m, n}^{k}$ is the relative propagation delay corresponding to the $m$ th transmitter and $n$th receiver with respect to the $k$ th scatterer, which is defined as

$$
\tau_{m, n}^{k}=\frac{1}{c}\left(\left|\mathbf{r}_{k}-\mathbf{R}_{t, m}\right|+\left|\mathbf{r}_{k}-\mathbf{R}_{r, n}\right|\right)
$$

where $\mathbf{R}_{t, m}$ and $\mathbf{R}_{r, n}$ are the position vectors of the $m$ th transmitter and $n$th receiver, respectively. $c$ is the speed of wave propagation.

For the sake of simplicity, the RCI formula needs a detecting signal [1], which can be simply structured as

$$
S_{n}\left(t, \mathbf{r}_{k}\right)=\sum_{m=1}^{M_{T}} S t_{m}\left(t-\tau_{m, n}^{k}\right) .
$$

Then, the echo can be expressed as the superposition of the detecting signals

$$
y_{n}(t)=\sum_{k \in S} \beta_{k} S_{n}\left(t, \mathbf{r}_{k}\right)
$$

Sample the echo and obtain $N$ samples; then we stack $\left\{y_{n}\left(t_{1}\right), \ldots, y_{n}\left(t_{N}\right)\right\}_{n=1}^{M_{R}}$ and $\left\{w_{n}\left(t_{1}\right), \ldots, w_{n}\left(t_{N}\right)\right\}_{n=1}^{M_{R}}$, via the vectorization operation $\operatorname{vec}(\cdot)$, into $M_{R} N$ dimensional column vectors $\mathbf{y}$ and $\mathbf{w}$, respectively. And

$$
\mathbf{s}\left(\mathbf{r}_{k}\right)=\operatorname{vec}\left(S_{n}\left(t, \mathbf{r}_{k}\right)\right)_{M_{R} N \times 1} .
$$

The column vector $\mathbf{s}\left(\mathbf{r}_{k}\right)$ is the atom corresponding to the $k$ th grid-cell. Then we arrange $\left\{\mathbf{s}\left(\mathbf{r}_{k}\right)\right\}_{k=1}^{K}$ into an $M_{R} N \times K$ dimensional matrix $\mathbf{S}=\left[\begin{array}{lllll}\mathbf{s}\left(\mathbf{r}_{1}\right) & \mathbf{s}\left(\mathbf{r}_{2}\right) & \cdots & \mathbf{s}\left(\mathbf{r}_{K}\right)\end{array}\right]$, which is often referred to as a dictionary matrix and defines the basic elements of sparse representation.

Denote by $\boldsymbol{\beta}=\left[\beta_{1}, \ldots, \beta_{K}\right]^{T}$ an unknown vector to be recovered. Then the measurement model reduces to (6), which is a familiar linear model used in most applications of sparse modeling:

$$
\mathbf{y}=\mathbf{S} \cdot \boldsymbol{\beta}+\mathbf{w}
$$

$\mathbf{S}$ can be deduced from the prior knowledge of the measurement model. Although the scattering coefficient vector $\boldsymbol{\beta}$ can be derived via the correlation between the echo and dictionary matrix directly, the sparse reconstruction methods are preferred to improve the resolution.

2.2. Off-Grid RCI Model. The imaging equation presented in (6) is derived under the grid matching condition. However, in most cases of radar imaging, the scatterers are distributed in a continuous scene. Regardless of how finely the imaging scene is discretized, the scatterers may not lie at the grid-cell centers, and then the off-grid problem yields. Considering the off-grid problem, the actual location of the $k$ th scatterer $\overline{\mathbf{r}}_{k}$ can be expressed as

$$
\widehat{\mathbf{r}}_{k}=\left(\bar{\theta}_{k}, \widehat{R}_{k}\right)=\mathbf{r}_{k}+\Delta \mathbf{r}_{k}
$$

where $\Delta \mathbf{r}_{k}=\left(\delta_{\theta_{k}} \Delta_{\theta}, \delta_{R_{k}} \Delta_{R}\right)$ is the OGE. $\left\{\delta_{\theta_{k}}, \delta_{R_{k}}\right\}$ denotes the normalized OGE which varies from -0.5 to 0.5 .

The off-grid effect can be compensated by estimating the perturbation $\left\{\delta_{\theta_{k}}, \delta_{R_{k}}\right\}$. In other words, the off-grid RCI is a joint estimation problem to recover $\left\{\beta_{k}, \theta_{k}, \delta_{\theta_{k}}, R_{k}, \delta_{R_{k}}\right\}_{k=1}^{K}$ associated with the nonzero $\beta_{k}$. As the estimation of $\left\{\delta_{\theta_{k}}, \delta_{R_{k}}\right\}$ is a nonlinear optimization problem which is analytically 




Observed variables

Unobserved variables

Parameters

FIgURE 2: A graphical representation of the model priors.

intractable, linearization through Taylor expansion is commonly used. Thus the true unknown dictionary is approximated, and then an accurate linear model for sparse reconstruction is established to reduce the OGE in the conventional fixed-dictionary setting.

Since the OGE is restricted in one grid-cell which is significantly small, then we make the following approximation by the first-order Taylor expansion:

$$
\begin{aligned}
\mathbf{s}\left(\widehat{\mathbf{r}}_{k}\right) \approx & \mathbf{s}\left(\mathbf{r}_{k}\right)+\left.\frac{\partial \mathbf{s}\left(\mathbf{r}_{k}\right)}{\partial \theta}\right|_{\theta=\theta_{k}} \Delta_{\theta} \delta_{\theta_{k}} \\
& +\left.\frac{\partial \mathbf{s}\left(\mathbf{r}_{k}\right)}{\partial R}\right|_{R=R_{k}} \Delta_{R} \delta_{R_{k}} .
\end{aligned}
$$

Denote $\boldsymbol{\delta}_{\theta}=\left[\delta_{\theta_{1}}, \ldots, \delta_{\theta_{K}}\right]^{T} \in \mathbb{R}^{K \times 1}, \boldsymbol{\delta}_{R}=\left[\delta_{R_{1}}, \ldots, \delta_{R_{K}}\right]^{T} \epsilon$ $\mathbb{R}^{K \times 1}, \mathbf{s}_{\theta}=\left[\left.\left(\partial \mathbf{s}\left(\mathbf{r}_{1}\right) / \partial \theta\right)\right|_{\theta=\theta_{1}},\left.\left(\partial \mathbf{s}\left(\mathbf{r}_{2}\right) / \partial \theta\right)\right|_{\theta=\theta_{2}}, \ldots,\left(\partial \mathbf{s}\left(\mathbf{r}_{K}\right) /\right.\right.$ $\left.\partial \theta)\left.\right|_{\theta=\theta_{K}}\right] \Delta_{\theta} \in \mathbb{C}^{M_{R} N \times K}$, and $\mathbf{S}_{R}=\left[\left.\left(\partial \mathbf{s}\left(\mathbf{r}_{1}\right) / \partial R\right)\right|_{R=R_{1}}\right.$, $\left.\left.\left(\partial \mathbf{s}\left(\mathbf{r}_{2}\right) / \partial R\right)\right|_{R=R_{2}}, \ldots,\left.\left(\partial \mathbf{s}\left(\mathbf{r}_{K}\right) / \partial R\right)\right|_{R=R_{K}}\right] \Delta_{R} \in \mathbb{C}^{M_{R} N \times K}$. The model in (6) could be concisely rewritten as

$$
\mathbf{y} \approx \mathbf{S} \cdot \boldsymbol{\beta}+\mathbf{S}_{\theta} \cdot\left(\boldsymbol{\delta}_{\theta} \odot \boldsymbol{\beta}\right)+\mathbf{S}_{R} \cdot\left(\boldsymbol{\delta}_{R} \odot \boldsymbol{\beta}\right)+\mathbf{w} .
$$

Alternatively, (9) can be expressed as

$$
\mathbf{y} \approx \mathbf{S} \cdot \boldsymbol{\beta}+\mathbf{S}_{\theta} \cdot \boldsymbol{\beta}_{\theta}+\mathbf{S}_{R} \cdot \boldsymbol{\beta}_{R}+\mathbf{w}
$$

where $\boldsymbol{\beta}_{\theta}=\boldsymbol{\delta}_{\theta} \odot \boldsymbol{\beta}$ and $\boldsymbol{\beta}_{R}=\boldsymbol{\delta}_{R} \odot \boldsymbol{\beta}$. Clearly, $\boldsymbol{\beta}_{\theta}$ and $\boldsymbol{\beta}_{R}$ are also sparse vectors and share the same support with $\boldsymbol{\beta}$. Hence, (10) means that the imaging equation can be sparsely approximated using three known dictionaries $\left\{\mathbf{S}, \mathbf{S}_{\theta}, \mathbf{S}_{R}\right\}$. Now, the problem of reconstructing the target image becomes recovering $\boldsymbol{\beta}, \boldsymbol{\beta}_{\theta}$, and $\boldsymbol{\beta}_{R}$ simultaneously based on (10). We approach this joint sparse recovery problem under the VSBL framework.

\section{Target Reconstruction: Principle and Algorithm}

The key to reconstruct the target image successfully is that all scatterers fall on the prediscretized grid-cell centers.
However, the generally existing off-grid problem jeopardizes the reconstruction performance seriously. In this section, VSBL is modified and then applied to off-grid RCI by reconstructing $\boldsymbol{\beta}, \boldsymbol{\beta}_{\theta}$, and $\boldsymbol{\beta}_{R}$ sequentially. This modified algorithm alleviates the off-grid effect and realizes the grid matching which means that the scatterers falling off the gridcell centers are captured by the closest grid-cell centers.

3.1. Two-Layer Hierarchical Prior Model. We begin with the specification of the graphical representation of model priors for model (10), as depicted in Figure 2.

To recover $\boldsymbol{\beta}, \boldsymbol{\beta}_{\theta}$, and $\boldsymbol{\beta}_{R}$ in (10), we model them as hidden variables and assign them appropriate priors. Typically, a two-level hierarchical Gaussian-Gamma prior is imposed on sparse coefficients to induce sparsity and also allows for a convenient conjugate-exponential analysis. Thus the following hierarchical prior is assigned:

$$
\begin{aligned}
p(\boldsymbol{\beta} \mid \boldsymbol{\gamma}) & =\mathscr{C} \mathcal{N}\left(\boldsymbol{\beta} \mid \mathbf{0}, \boldsymbol{\Lambda}^{-1}\right) \\
& =\frac{1}{\pi^{K} \operatorname{det}(\boldsymbol{\Lambda})^{-1}} \exp \left\{-\boldsymbol{\beta}^{H} \boldsymbol{\Lambda} \boldsymbol{\beta}\right\}, \\
p(\boldsymbol{\gamma} ; a, b) & =\prod_{k=1}^{K} \Gamma\left(\gamma_{k} \mid a, b\right),
\end{aligned}
$$

where $\mathscr{C} \mathscr{N}(\cdot \mid \boldsymbol{\mu}, \boldsymbol{\Sigma})$ denotes the complex Gaussian distribution with mean vector $\boldsymbol{\mu}$ and covariance matrix $\boldsymbol{\Sigma}$. Consider $\gamma=\left[\gamma_{1}, \gamma_{2}, \ldots, \gamma_{K}\right]^{T}, \Lambda=\operatorname{diag}(\gamma), \gamma_{k}^{-1}$ is the prior variance of $\beta_{k}$, and $\Gamma\left(\gamma_{k} \mid a, b\right)$ is the Gamma distribution with parameters $a$ and $b$. The prior parameters $\gamma$ are also called sparsity parameters, being inversely proportional to the width of the pdf. Naturally, a large value of $\gamma_{k}$ will drive the corresponding weight $\beta_{k}$ to zero, thus inducing a sparse solution. As $\left\{\delta_{\theta_{k}}, \delta_{R_{k}}\right\} \in[-0.5,0.5], \beta_{\theta_{k}}=\beta_{k} \delta_{\theta_{k}}, \beta_{R_{k}}=\beta_{k} \delta_{R_{k}}$, and the prior variance of $\beta_{k}$ is $\gamma_{k}^{-1}$; then the prior variances of $\beta_{\theta_{k}}$ and $\beta_{R_{k}}$ can be set to be $\gamma_{k}^{-1} / 4$ to accommodate the worst case, where the true scatterer is at the middle of the two consecutive grids. Besides, this setting satisfies the constraint of common support among $\boldsymbol{\beta}_{\theta}, \boldsymbol{\beta}_{R}$, and $\boldsymbol{\beta}$. Consequently, the priors of $\boldsymbol{\beta}_{\theta}$ and $\boldsymbol{\beta}_{R}$ conditioned on $\boldsymbol{\gamma}$ can be expressed as $\mathscr{C} \mathscr{N}\left(\boldsymbol{\beta}_{\theta}\right.$ $\left.\mathbf{0},(1 / 4) \Lambda^{-1}\right)$ and $\mathscr{C} \mathcal{N}\left(\boldsymbol{\beta}_{R} \mid \mathbf{0},(1 / 4) \Lambda^{-1}\right)$, respectively. 
In addition, the measurement noise is assumed to be independent and complex Gaussian with zero-mean and variance $\gamma_{0}^{-1}$, to allow conjugate-exponential analysis:

$$
\begin{aligned}
p\left(\mathbf{w} \mid \gamma_{0}\right) & =\mathscr{C} \mathcal{N}\left(\mathbf{w} \mid \mathbf{0}, \gamma_{0}^{-1} \mathbf{I}\right) \\
& =\left(\pi \gamma_{0}^{-1}\right)^{-K} \exp \left\{-\gamma_{0}\|\mathbf{w}\|_{2}^{2}\right\}, \\
p\left(\gamma_{0} ; c, d\right) & =\Gamma\left(\gamma_{0} \mid c, d\right) .
\end{aligned}
$$

3.2. Off-Grid Variational Sparse Bayesian Learning. Based on the model presented above, (10) becomes a model containing only hidden variables and no parameters. Then we employ the SBL to realize the target reconstruction. However, the derivation of the posterior $p(\mathscr{H} \mid \mathbf{y})$ is generally intractable in the SBL framework. Thus VBI is adopted in this case to obtain the variational solution to SBL.

VBI is a methodology that can deal with complicated Bayesian models [23], aiming at finding maximum likelihood estimates of unknown parameters involved in a Bayesian model with hidden (i.e., unobserved) variables. VBI first uses variational methods to find approximate posterior distributions of hidden variables and then updates the estimates of unknown parameters based on the posterior densities of hidden variables. Specifically, the variational solution is obtained by maximizing a variational lower bound on a logevidence $\ln p(\mathbf{y})[25,26]$, which is equivalent to minimize the Kullback-Leibler divergence (KLD) between the approximating pdf $q(\mathscr{H})$ and the posterior pdf $p(\mathscr{H} \mid$ y). Moreover, a structured mean field approximation can be applied to compute the approximate posterior $q(\mathscr{H})$ :

$$
q(\mathscr{H})=q(\boldsymbol{\beta}) q\left(\boldsymbol{\beta}_{\theta}\right) q\left(\boldsymbol{\beta}_{R}\right) q(\boldsymbol{\gamma}) q\left(\gamma_{0}\right),
$$

where $q(\gamma)=\prod_{k=1}^{K} q\left(\gamma_{k}\right)$ automatically follows from the independence assumption (pdf factorization) among $\boldsymbol{\beta}, \boldsymbol{\beta}_{\theta}$, $\boldsymbol{\beta}_{R}$, and $\boldsymbol{\gamma}$ posterior approximations. Meanwhile, VBI obtains the posterior densities via [24]

$$
\ln q_{k}=\langle\ln p(\mathbf{y}, \mathscr{H})\rangle_{i \neq k}+\text { const }
$$

where $\mathscr{H}=\left\{\boldsymbol{\beta}, \boldsymbol{\beta}_{\theta}, \boldsymbol{\beta}_{R}, \boldsymbol{\gamma}, \gamma_{0}\right\}$ is the set of hidden variables, $q_{k}$ denotes the posterior density for $\mathscr{H}_{k}, p(\mathbf{y}, \mathscr{H})$ is the joint pdf defined as $p(\mathbf{y}, \mathscr{H})=p\left(\mathbf{y} \mid \boldsymbol{\beta}, \boldsymbol{\beta}_{\theta}, \boldsymbol{\beta}_{R}, \gamma_{0}\right) p(\boldsymbol{\beta} \mid \boldsymbol{\gamma}) p\left(\boldsymbol{\beta}_{\theta} \mid\right.$ ү) $p\left(\boldsymbol{\beta}_{R} \mid \boldsymbol{\gamma}\right) p(\boldsymbol{\gamma} ; a, b) p\left(\gamma_{0} ; c, d\right),\langle\cdot\rangle_{i \neq k}$ denotes the expectation with respect to $q_{i}$, and const is a normalized constant.

By using (14), the variational approximating factors of $q(\mathscr{H})$ can be obtained as $q(\boldsymbol{\beta})=\mathscr{C} \mathscr{N}\left(\boldsymbol{\beta} \mid \boldsymbol{\mu}_{\boldsymbol{\beta}}, \boldsymbol{\Sigma}_{\boldsymbol{\beta}}\right), q\left(\boldsymbol{\beta}_{\theta}\right)=$ $\mathscr{C} \mathcal{N}\left(\boldsymbol{\beta}_{\theta} \mid \boldsymbol{\mu}_{\boldsymbol{\beta}_{\theta}}, \boldsymbol{\Sigma}_{\boldsymbol{\beta}_{\theta}}\right), q\left(\boldsymbol{\beta}_{R}\right)=\mathscr{C} \mathscr{N}\left(\boldsymbol{\beta}_{R} \mid \boldsymbol{\mu}_{\boldsymbol{\beta}_{R}}, \boldsymbol{\Sigma}_{\boldsymbol{\beta}_{R}}\right), q\left(\gamma_{k}\right)=$ $\operatorname{Gamma}\left(\gamma_{k} \mid \bar{a}, \bar{b}_{k}\right)$, and $q\left(\gamma_{0}\right)=\operatorname{Gamma}\left(\gamma_{0} \mid \bar{c}, \bar{d}\right)$. The parameters of the approximating factors, that is, variational parameters, can be computed as follows:

$$
\begin{aligned}
& \boldsymbol{\Sigma}_{\boldsymbol{\beta}}=\left(\widehat{\gamma}_{0} \mathbf{S}^{H} \mathbf{S}+\bar{\Lambda}\right)^{-1}, \\
& \boldsymbol{\mu}_{\boldsymbol{\beta}}=\widehat{\gamma}_{0} \boldsymbol{\Sigma}_{\boldsymbol{\beta}} \mathbf{S}^{H}\left(\mathbf{y}-\mathbf{S}_{\theta} \boldsymbol{\mu}_{\boldsymbol{\beta}_{\theta}}-\mathbf{S}_{R} \boldsymbol{\mu}_{\boldsymbol{\beta}_{R}}\right),
\end{aligned}
$$

$$
\begin{aligned}
\boldsymbol{\Sigma}_{\boldsymbol{\beta}_{\theta}}= & \left(\widehat{\gamma}_{0} \mathbf{S}_{\theta}^{H} \mathbf{S}_{\theta}+4 \overline{\boldsymbol{\Lambda}}\right)^{-1}, \\
\boldsymbol{\mu}_{\boldsymbol{\beta}_{\theta}}= & \widehat{\gamma}_{0} \boldsymbol{\Sigma}_{\boldsymbol{\beta}_{\theta}} \mathbf{S}_{\theta}^{H}\left(\mathbf{y}-\mathbf{S} \boldsymbol{\mu}_{\boldsymbol{\beta}}-\mathbf{S}_{R} \boldsymbol{\mu}_{\boldsymbol{\beta}_{R}}\right) \\
\boldsymbol{\Sigma}_{\boldsymbol{\beta}_{R}}= & \left(\widehat{\gamma}_{0} \mathbf{S}_{R}^{H} \mathbf{S}_{R}+4 \overline{\boldsymbol{\Lambda}}\right)^{-1} \\
\boldsymbol{\mu}_{\boldsymbol{\beta}_{R}=}= & \widehat{\gamma}_{0} \boldsymbol{\Sigma}_{\boldsymbol{\beta}_{R}} \mathbf{S}_{R}^{H}\left(\mathbf{y}-\mathbf{S} \boldsymbol{\mu}_{\boldsymbol{\beta}}-\mathbf{S}_{\theta} \boldsymbol{\mu}_{\boldsymbol{\beta}_{\theta}}\right) \\
\bar{a}= & a+3 \\
\bar{b}_{k}= & b+\left\{\left[\boldsymbol{\Sigma}_{\boldsymbol{\beta}}\right]_{k k}+\left|\left[\boldsymbol{\mu}_{\boldsymbol{\beta}}\right]_{k}\right|^{2}\right\} \\
& +4\left\{\left[\boldsymbol{\Sigma}_{\boldsymbol{\beta}_{\theta}}\right]_{k k}+\left|\left[\boldsymbol{\mu}_{\boldsymbol{\beta}_{\theta}}\right]_{k}\right|^{2}\right\} \\
& +4\left\{\left[\boldsymbol{\Sigma}_{\boldsymbol{\beta}_{R}}\right]_{k k}+\left|\left[\boldsymbol{\mu}_{\boldsymbol{\beta}_{R}}\right]_{k}\right|^{2}\right\} \\
\bar{c}= & c+M_{R} N, \\
\bar{d}= & d+\left\|\mathbf{y}-\mathbf{S}_{\boldsymbol{\mu}_{\boldsymbol{\beta}}}-\mathbf{S}_{\theta} \boldsymbol{\mu}_{\boldsymbol{\beta}_{\theta}}-\mathbf{S}_{R} \boldsymbol{\mu}_{\boldsymbol{\beta}_{R}}\right\|_{2}^{2}+\widehat{\gamma}_{0}^{-1} \sum_{k=1}^{K} \lambda_{k}
\end{aligned}
$$

where $\lambda_{k}=3-\bar{\gamma}_{k}\left\{\left[\boldsymbol{\Sigma}_{\boldsymbol{\beta}}\right]_{k k}+4\left[\boldsymbol{\Sigma}_{\boldsymbol{\beta}_{\theta}}\right]_{k k}+4\left[\boldsymbol{\Sigma}_{\boldsymbol{\beta}_{R}}\right]_{k k}\right\}, \overline{\boldsymbol{\Lambda}}=$ $\operatorname{diag}\left(\bar{\gamma}_{1}, \bar{\gamma}_{2}, \ldots, \bar{\gamma}_{K}\right),\langle\boldsymbol{\beta}\rangle=\boldsymbol{\mu}_{\boldsymbol{\beta}},\left\langle\boldsymbol{\beta}_{\theta}\right\rangle=\boldsymbol{\mu}_{\boldsymbol{\beta}_{\theta}},\left\langle\boldsymbol{\beta}_{R}\right\rangle=\boldsymbol{\mu}_{\boldsymbol{\beta}_{R}},[\cdot]_{k k}$ represents the $k$ th main diagonal element of a matrix, and $[\cdot]_{k}$ is the $k$ th element of a vector. Using the property of Gamma distribution, the expected values can be computed as

$$
\begin{aligned}
& \bar{\gamma}_{k}=\frac{\bar{a}}{\bar{b}_{k}}, \\
& \bar{\gamma}_{0}=\frac{\bar{c}}{\bar{d}} .
\end{aligned}
$$

Based on the above results, the posterior distributions of $\mathscr{H}$ can be obtained by iteratively performing (15)-(20) until convergence. During that process, the parameters $a, b$, $c$, and $d$ are typically fixed at values close to zero to assume noninformative priors for $\gamma$ and $\gamma_{0}$. Alternatively, the update for $\bar{\gamma}_{k}$ and $\bar{\gamma}_{0}$ can be changed as follows:

$$
\begin{aligned}
& \bar{\gamma}_{k}=\frac{a+\lambda_{k}}{b+\left|\boldsymbol{\mu}_{\boldsymbol{\beta}, k}\right|^{2}+4\left|\boldsymbol{\mu}_{\boldsymbol{\beta}_{\theta}, k}\right|^{2}+4\left|\boldsymbol{\mu}_{\boldsymbol{\beta}_{R}, k}\right|^{2}}, \\
& \bar{\gamma}_{0}=\frac{c+M_{R} N-\sum_{k=1}^{K} \lambda_{k}}{d+\| \mathbf{y}-\mathbf{S} \boldsymbol{\mu}_{\boldsymbol{\beta}}-\mathbf{S}_{\theta} \boldsymbol{\mu}_{\boldsymbol{\beta}_{\theta}}-\mathbf{S}_{R} \boldsymbol{\mu}_{\boldsymbol{\beta}_{R} \|_{2}^{2}}} .
\end{aligned}
$$

The updates in (21) are observed to lead to much faster convergence although they do not benefit from the guarantee of local optimum of object function. It is remarkable that each $\lambda_{k} \in[0,3]$ can be interpreted as a measure of how "welldetermined" its corresponding parameters $\beta_{k}, \beta_{\theta_{k}}$, and $\beta_{R_{k}}$ are by the data. When $\gamma_{k}$ is large, where $\beta_{k}, \beta_{\theta_{k}}$, and $\beta_{R_{k}}$ are highly constrained by the prior, $\left[\Sigma_{\beta}\right]_{k k}+4\left[\Sigma_{\beta_{\theta}}\right]_{k k}+4\left[\Sigma_{\beta_{R}}\right]_{k k} \approx$ $3 / \gamma_{k}$ and it follows that $\lambda_{k} \approx 0$; otherwise, $\beta_{k}, \beta_{\theta_{k}}$, and $\beta_{R_{k}}$ fit the data, $\lambda_{k} \approx 3$.

Due to the convexity of the variational lower bound in the approximating factors $q(\boldsymbol{\beta}), q\left(\boldsymbol{\beta}_{\theta}\right), q\left(\boldsymbol{\beta}_{R}\right), q\left(\gamma_{k}\right)$, and $q\left(\gamma_{0}\right)$, we 




Algorithm 1: OG-VSBL algorithm.

can update these factors in any order while keeping the other factors fixed. However, the update order is important since different update orders might lead to different local optima of the variational lower bound.

In addition, we terminate the algorithm if $\| \bar{\gamma}^{i+1}-$ $\bar{\gamma}^{i}\left\|_{2} /\right\| \bar{\gamma}^{i} \|_{2}<\eta$ or the maximum number of iterations $I_{\max }$ is reached, where $\eta$ is a user-defined tolerance and the superscript $i$ refers to the iteration.

3.3. Fast Implementation. The above algorithm suffers from two main drawbacks: high computational complexity and low convergence rate. As each iteration consists of matrix inversion and matrix-vector multiplication in (15)-(17), the costs of the two operations are $o\left(K^{3}\right)$ and $o\left(K^{2}\right)$, respectively. Hence, the procedure is computationally expensive to implement when $K$ is large. This exists generally since a dense grid is required in principle to reduce the error incurred by the linear approximation. In order to overcome the drawbacks, a fast implementing approach is developed.

In conventional SBL, many of the prior precisions of coefficients typically take on quite large values upon convergence. This implies that the corresponding coefficients are quite small and in turn the contributions of the corresponding atoms are negligible; thus these atoms can be pruned reasonably to realize sparsity [27]. Then we maintain this feature in the algorithm described above.

From (15)-(17), it can be shown that when $\gamma_{k}$ is large enough, $\left[\boldsymbol{\Sigma}_{\boldsymbol{\beta}}\right]_{k k},\left[\boldsymbol{\Sigma}_{\boldsymbol{\beta}_{\theta}}\right]_{k k},\left[\boldsymbol{\Sigma}_{\boldsymbol{\beta}_{R}}\right]_{k k},\left[\boldsymbol{\mu}_{\boldsymbol{\beta}}\right]_{k},\left[\boldsymbol{\mu}_{\boldsymbol{\beta}_{\theta}}\right]_{k}$, and $\left[\boldsymbol{\mu}_{\boldsymbol{\beta}_{R}}\right]_{k}$ will be close to 0 , which means that the contribution of the $k$ th grid to signal synthesis can be neglected, and thus this grid could be removed from the current grid set. Based on this observation, grid pruning can be conducted through the threshold of $\gamma_{k}$. Specifically, denoting a large threshold $\gamma_{\text {th }}$, the pruning of the current grid set $S_{\text {grid }}^{i}$ can be achieved via

$$
S_{\text {grid }}^{i+1}=\left\{k \mid \bar{\gamma}_{k}<\gamma_{\text {th }}, k \in S_{\text {grid }}^{i}\right\},
$$

where $S_{\text {grid }}^{i+1}$ denotes the grid set after pruning. It should be mentioned that, due to the grid pruning, the posterior mean vectors $\left(\boldsymbol{\mu}_{\boldsymbol{\beta}}, \boldsymbol{\mu}_{\boldsymbol{\beta}_{\theta}}\right.$, and $\left.\boldsymbol{\mu}_{\boldsymbol{\beta}_{R}}\right)$, covariance matrix $\left(\boldsymbol{\Sigma}_{\boldsymbol{\beta}}, \boldsymbol{\Sigma}_{\boldsymbol{\beta}_{\theta}}\right.$, and $\Sigma_{\beta_{R}}$ ), and the dictionaries $\left(\mathbf{S}, \mathbf{S}_{\theta}\right.$, and $\mathbf{S}_{R}$ ) should be also pruned accordingly.

Because of the grid pruning, the computational burden is reduced and the convergence speed is improved, the sparsity of the reconstruction results is enhanced, and meanwhile the common support shared by the scattering coefficients and OGE is guaranteed. Thus the reconstruction performance would be better, whereas the threshold $\gamma_{\text {th }}$ needs to be selected carefully; otherwise the grid would be pruned mistakenly.

3.4. Algorithm Summarization. Based on the above discussions, the procedure of the proposed algorithm, that is, offgrid variational sparse Bayesian learning (OG-VSBL), is given in Algorithm 1.

Next, we provide the following comments to provide further insight into the proposed algorithm.

(1) The Advantages. Similar to other sparse-based or CSbased algorithms, the OG-VSBL algorithm performs well for high-resolution RCI under the scenarios of low signalto-noise ratio (SNR) with only a few measurements. While compared with many sparse-based algorithms, the proposed algorithm is a data-driven learning process which needs none of the parameters tuned manually. Furthermore, the OGVSBL algorithm utilizes the hierarchical modeling procedure to encode signal sparsity which could obtain the approximate posterior distribution and is regarded as a full Bayesian method. The statistical information is used to enhance the estimation performance and avoid converging to a shallow local minimum during the learning procedure, due to the utilization of higher order statistical information.

(2) Comparison with Other Algorithms. As discussed in Section 1, various methods could solve the off-grid problem, such as band-excluded locally optimized orthogonal matching pursuit (BLOOMP) [17], JCP [3], S-TLS [18], and block sparse Bayesian learning (BSBL) [21]. As a greedy algorithm, BLOOMP modifies the search strategy of OMP and improves the performance of OMP in the presence of offgrid scatterers. JCP models the off-grid imaging equation as $\mathbf{y}=\mathbf{S} \cdot \boldsymbol{\beta}+\boldsymbol{\varepsilon}+\mathbf{w}$, where $\boldsymbol{\varepsilon} \approx \mathbf{S}_{\theta} \cdot \boldsymbol{\beta}_{\theta}+\mathbf{S}_{R} \cdot \boldsymbol{\beta}_{R}$ denotes the modeling error caused by off-grid effect. Then JCP uses the correlation method to estimate the modeling error and then iteratively modifies the results of the parameterized method. STLS seeks to solve the nonconvex optimization problem:

$$
\min _{\boldsymbol{\beta}, \mathbf{E}}\left\{\|\mathbf{y}-(\mathbf{S}+\mathbf{E}) \boldsymbol{\beta}\|_{2}^{2}+\|\mathbf{E}\|_{F}^{2}+\lambda\|\boldsymbol{\beta}\|_{1}\right\}
$$


where $\lambda$ is a regularization parameter and $\mathbf{E}$ denotes the dictionary perturbation caused by off-grid effect. From the Bayesian perspective, the S-TLS yield an MAP solution if $\mathbf{E}$ is Gaussian which cannot be satisfied in the off-grid RCI. These algorithms are designed for RCI with modeling error (including OGE), but they do not consider the structure of the dictionary perturbation from statistic viewpoint. It is noteworthy that $\boldsymbol{\beta}_{\theta}$ and $\boldsymbol{\beta}_{R}$ share the same support with $\boldsymbol{\beta}$; thus the unknown variables $\left\{\boldsymbol{\beta}, \boldsymbol{\beta}_{\theta}, \boldsymbol{\beta}_{R}\right\}$ can regroup into $K$ nonoverlapping subblocks and the $k$ th subblock is $\mathbf{x}_{k}=$ $\left[\beta, \beta_{\theta_{k}}, \beta_{R_{k}}\right]^{T}$. Then $\mathbf{x}$ is block sparse and can be solved in BSBL framework. Both BSBL and OG-VSBL formulate the off-grid RCI using Bayesian hierarchical prior modeling, but their implementation processes are different.

\section{Discussion and Numerical Analysis}

In this section, the performance of the proposed off-grid RCI algorithm is analyzed by conducting a wide range of numerical experiments. The imaging performance is indicated by two criteria, that is, the reconstructed sparsity $\|\overline{\boldsymbol{\beta}}\|_{0}$ and relative imaging error (RIE) which is expressed as $\xi=$ $20 \log _{10}\left(\|\overline{\boldsymbol{\beta}}-\boldsymbol{\beta}\|_{2} /\|\boldsymbol{\beta}\|_{2}\right)$.

Consider an X-band RCI system with carrier frequency of $10 \mathrm{GHz}$. Both the transmitters and receivers are configured as a uniform linear array (ULA) with the numbers of elements $M_{T}=M_{R}=8$ and interelement spacing $d_{T}=d_{R}=1 \mathrm{~m}$, respectively. The transmitters transmit frequency-hopping $(\mathrm{FH})$ waveforms, described in $[28,29]$, with the minimum $\mathrm{FH}$ interval $1 \mathrm{MHz}$ and maximal hopping frequency $500 \mathrm{MHz}$. The waveforms are comprised by 400 hopping frequencies with the FH duration $10 \mathrm{~ns}$. With the above parameters, the grid size $\left(\Delta_{\theta}, \Delta_{R}\right)$ for conventional imaging approaches can be computed, while we set a smaller grid size for the super-resolving ability of sparse reconstruction approaches. A range-azimuth target space, covering $4 \mathrm{~m} \times 0.04 \mathrm{rad}$, is discretized to $40 \times 40$ grid-cells. Further, there supposed to be six widely separated point scatterers which are randomly located within the grid-cells.

In the proposed OG-VSBL algorithm, we initialize $\gamma_{0}=$ $100 / \operatorname{var}(\mathbf{y}), \boldsymbol{\gamma}=\left|\mathbf{S}^{H} \mathbf{y}\right| /\left(M_{R} N\right), \boldsymbol{\beta}_{\theta}=\boldsymbol{\beta}_{R}=\mathbf{0}$, and $a=b=$ $c=d=10^{-6}$. For the termination condition, we set $\eta=10^{-3}$ and the maximum number of iterations to $I_{\max }=100$. The threshold for grid pruning is $\gamma_{\text {th }}=10^{4}$.

4.1. Performance of OG-VSBL. To illuminate the validity of the proposed OG-VSBL algorithm, we conduct a numerical simulation where VSBL algorithm is implemented as a comparison. Figure 3 shows the imaging results. The white circles in Figure 3(a) represent the actual locations of scatterers and the red points represent the grid-cells closest to the scatterers. In this example, the external noise is fixed at $\mathrm{SNR}=20 \mathrm{~dB}$. Figures 3(b) and 3(c) plot the reconstructed images with respect to on-grid and off-grid targets, respectively, where VSBL is utilized. When the scatterers fall on the grid-cell centers, VSBL can reconstruct the target perfectly, while for off-grid target, the image is defocused as shown in Figure 3(c), where many spurious scatterers exist and the signal energy spills over the imaging plane. Compared with Figure 3(c), the image shown in Figure 3(d) is improved significantly. Six scatterers are reconstructed much better without any obvious spurious scatterer, mainly as the prior knowledge of sparsity and the OGE are fully utilized. Thus OG-VSBL can be used to image the off-grid target.

Figure 4 depicts the RIEs for both the VSBL and OGVSBL decrease during the imaging process. Both algorithms are converged after about 20 iterations, indicating the validity of the algorithms. As the prior knowledge of both the sparsity and the distributions of scattering coefficient and the OGE are fully utilized, the OG-VSBL algorithm improves the imaging performance by about $8 \mathrm{~dB}$ from the RIE perspective, compared with VSBL.

As OG-VSBL is a sparse reconstruction-type algorithm whose performance depends on the sparse prior of target, we test the performance under the sparsity degree of target. In the experiment, OG-VSBL is implemented for different sparsity degrees varying from 5 to 35 , and VSBL is also simulated for comparison. For each sparsity degree, the scattering centers are randomly located within the grid-cells and the RIE and reconstructed sparsity are recorded over 100 independent trials.

Figure 5 shows the reconstructed results. It can be observed from Figure 5(a) that the performance lies in the sparsity property of a target for both algorithms and the performance degrades as the sparsity degree increases. Besides, OG-VSBL reconstructs the target precisely without spurious scatterer when the sparsity degree is not higher than 15 under the simulation parameters. For targets with higher sparsity degree, it is observed that OG-VSBL generates slightly more scatterers than the true sparsity degree but still obtains much sparser image than VSBL. As seen from Figure 5(b), the RIE reconstructed by OG-VSBL increases with the sparsity degree, which means the image quality tends to be worse. Then we can conclude that performance of OGVSBL is affected by the sparsity degree of the target, since it is based on the prior knowledge of sparsity.

4.2. Performance Comparison with Other Algorithms. In this subsection, the performance of OG-VSBL is compared with that of other algorithms which take into account off-grid effect. These algorithms for comparison include BLOOMP, JCP, S-TLS, and BSBL algorithms. All these algorithms realize the grid matching which means that the off-grid scatterers are captured by the closest grid-cell centers.

Figure 6 shows the original image and target images reconstructed by the five algorithms. For BLOOMP, JCP, and S-TLS, the reconstructed images are blurry and the signal energy spills over the target space as depicted in Figures 6(b)6(d). Apart from the six strong scatterers, many spurious scatterers exist around the actual scatterers, which will lead to failure in picking up the scatterers without other prior knowledge (i.e., the number and scattering coefficients of scatterers). Comparably, the image reconstructed by BSBL is much sparser as presented in Figure 6(e), but a few spurious scatterers still exist. In contrast with Figures 6(b)-6(e), the image shown in Figure 6(f) is improved significantly. Six 




(a)

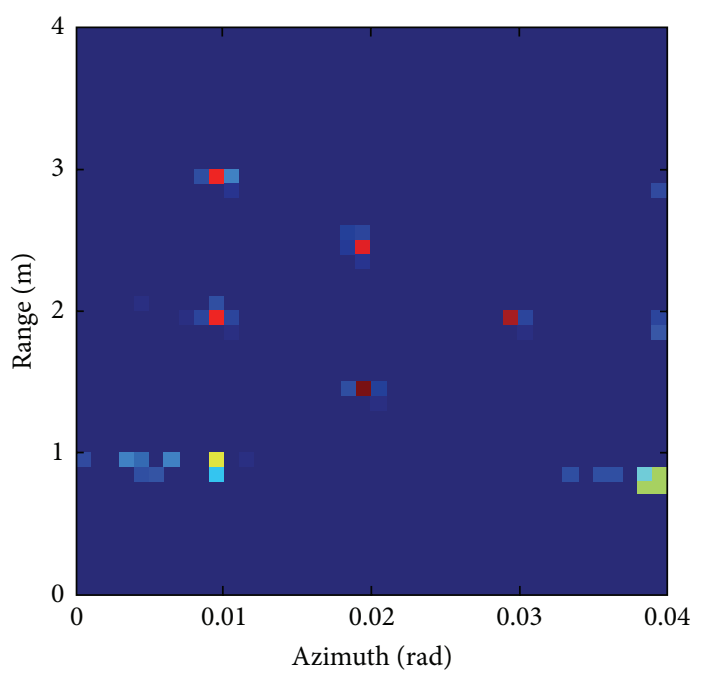

(c)

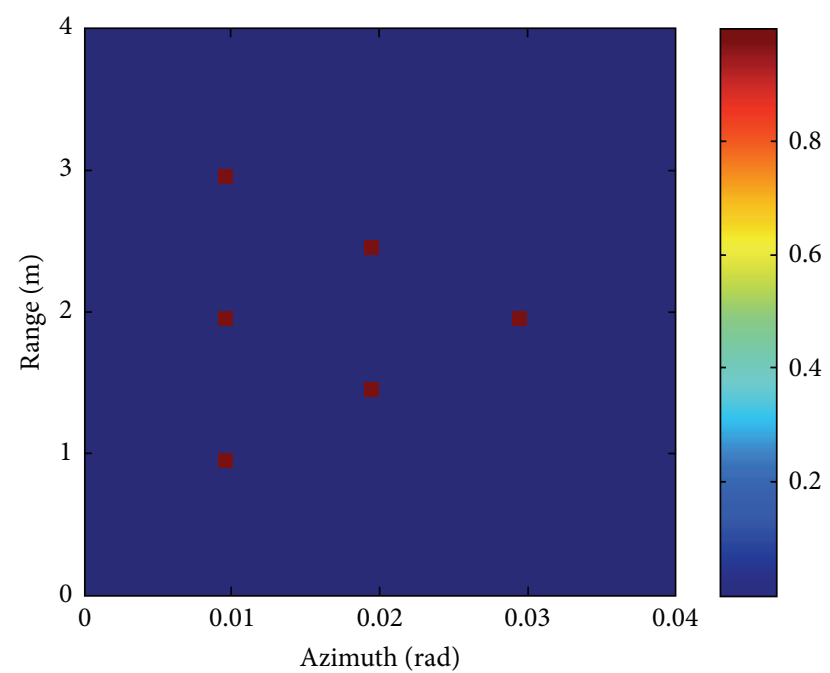

(b)

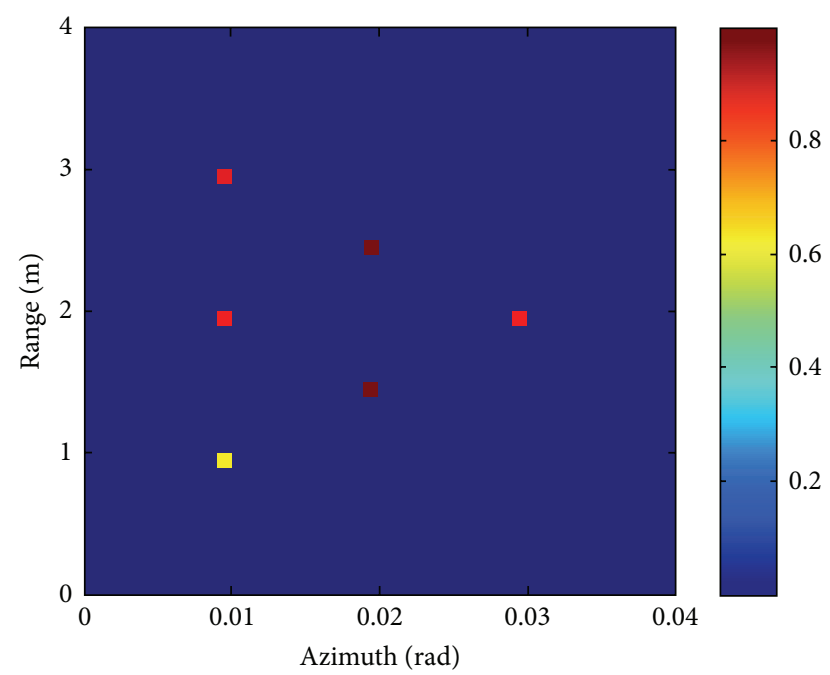

(d)

FIGURE 3: RCI results: (a) original image; (b) VSBL imaging result for on-grid target; (c) VSBL imaging result for off-grid target; (d) OG-VSBL imaging result for off-grid target.

scatterers are reconstructed much better without any obvious spurious scatterer, and their support is recovered precisely, mainly as the prior knowledge of sparsity and the OGE are fully exploited. Thus, for off-grid RCI, OG-VSBL shows the best performance among the algorithms tested above.

Next, we vary the SNR to evaluate the performance of OG-VSBL by numerical experiments. To provide the lower bound for the listed algorithms, we define the Oracle solution for grid matching, which is the least squares solution within the true support set [30]; that is,

$$
\begin{aligned}
& \left(\overline{\boldsymbol{\beta}}_{\mathrm{or}}\right)_{S}=\mathbf{S}_{S}^{\dagger} \mathbf{y}, \\
& \left(\overline{\boldsymbol{\beta}}_{\mathrm{or}}\right)_{S^{C}}=\mathbf{0},
\end{aligned}
$$

where $\mathbf{S}_{S}$ is the dictionary associated with the support set $S$ and $S^{C}$ is the complement of the support set $S$.
TABLE 1: Reconstructed sparsity for different algorithms with varying SNRs.

\begin{tabular}{lccccc}
\hline SNR $(\mathrm{dB})$ & BLOOMP & JCP & S-TLS & BSBL & OG-VSBL \\
\hline-20 & 30 & 90 & 18 & 28 & 8 \\
-10 & 30 & 32 & 21 & 12 & 8 \\
0 & 25 & 33 & 25 & 7 & 7 \\
10 & 25 & 33 & 25 & 7 & 6 \\
20 & 25 & 34 & 21 & 7 & 6 \\
30 & 25 & 35 & 22 & 7 & 6 \\
40 & 25 & 36 & 22 & 7 & 6 \\
\hline
\end{tabular}

The results are shown in Figure 7 and Table 1. Monte Carlo experiments are conducted to test the robustness of the listed algorithms to different SNRs varying from -20 to $40 \mathrm{~dB}$. For each SNR, we conduct 100 independent trials and record 


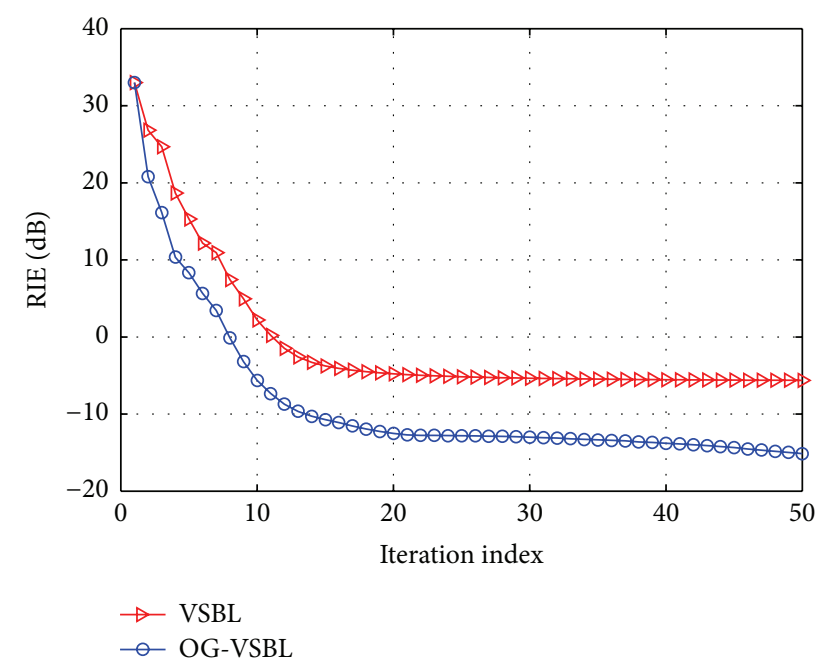

FIGURE 4: The convergence of the reconstructed scattering coefficients versus the number of iterations.

the RIE and reconstructed sparsity. Figure 7 shows the RIEs under different SNRs. It follows that as the SNR increases, the RIEs decrease quickly to an asymptotic region where the RIEs keep nearly unchanged, implying that the performance is improved and the algorithms are sensitive to noise in low SNR regimes, while, among the five algorithms, OG-VSBL shows the best performance. Besides, the RIE obtained by OG-VSBL is much closer to that obtained by Oracle solution and approaches the lower bound defined by Oracle estimator especially in high SNR regimes. The reconstructed sparsity is shown in Table 1. Algorithms like BLOOMP, JCP, and STLS tend to generate nonsparse and defocused images in the presence of off-grid scatterers, since they do not exploit the off-grid problem accurately. Therefore, the signal energy spills over the target space and the spurious scatterers emerge, especially in low SNR regimes. As presented in Table 1, BSBL and OG-VSBL can achieve much sparser image due to the utilization of the hierarchical Bayesian modeling; thus the image quality is improved significantly.

To make further comparisons, the performance of OGVSBL under different grid sizes is investigated, comparing with the other algorithms. In this experiment, we assume that the grid number and actual scatterer distribution are invariant; the discretized grid size is changed by grid refinement. The refinement factor $r$, defined as the proportion of the refinement grid size and initial grid size, is used as a metric of grid size. We assign SNR $=20 \mathrm{~dB}$ and conduct 50 independent trials to achieve the averaged RIEs.

Table 2 presents the simulation result where the RIE is shown to be sensitive to the refinement factor. It can be shown from Table 2 that the refinement factor $r=0.6$ induces the highest reconstruction error; all algorithms except JCP have the worst performance deterioration. As the refinement factor increases, the grid-cell becomes larger which yields a high coherence between the columns of the dictionary. Then the RIP condition for reliable sparse recovery is guaranteed, and the RIEs would be lower. When the refinement factor



(a)

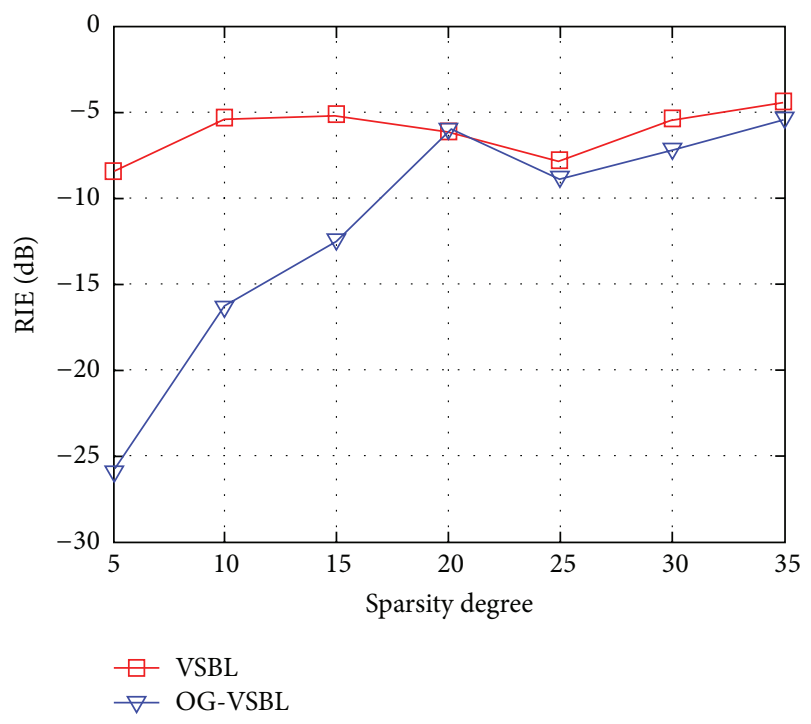

(b)

FIGURE 5: Performance under different sparsity degrees: (a) reconstructed sparsity versus sparsity degree; (b) RIE versus sparsity degree.

TABLE 2: RIEs (dB) for different algorithms with varying refinement factor.

\begin{tabular}{lcccccc}
\hline$r$ & BLOOMP & JCP & S-TLS & BSBL & OG-VSBL & Oracle \\
\hline 1 & -5.4 & -2.5 & -2.1 & -13 & -14.5 & -16.6 \\
0.8 & -4.9 & 20.1 & -2.4 & -9.6 & -7.5 & -20.2 \\
0.6 & -1.2 & 85.5 & -0.2 & -3.8 & -8.6 & -21.8 \\
0.4 & -4.7 & 154.0 & 0.2 & -5.7 & -7.5 & -31.7 \\
0.2 & -6.6 & 172.8 & 1.3 & -12.8 & -14.7 & -43.4 \\
\hline
\end{tabular}

becomes smaller, the RIEs also decrease because the modeling error caused by off-grid problem decreases accordingly, which is indicated by the Oracle solution. Furthermore, a smaller grid size would decrease the approximation error 


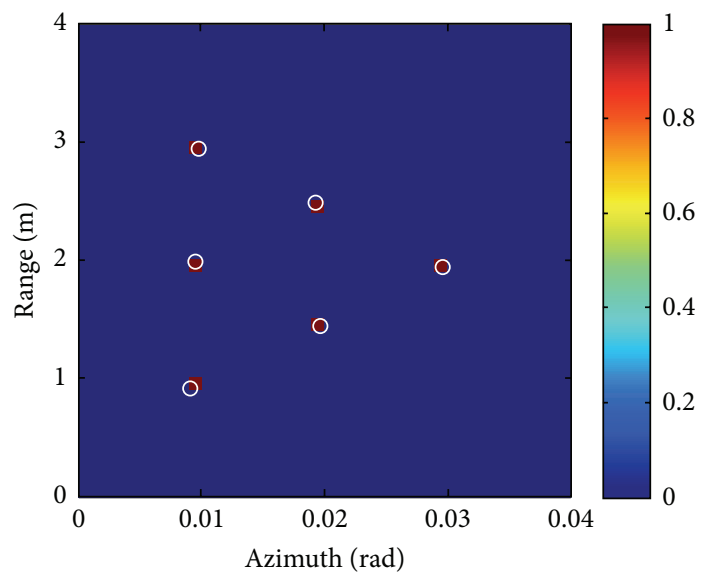

(a)

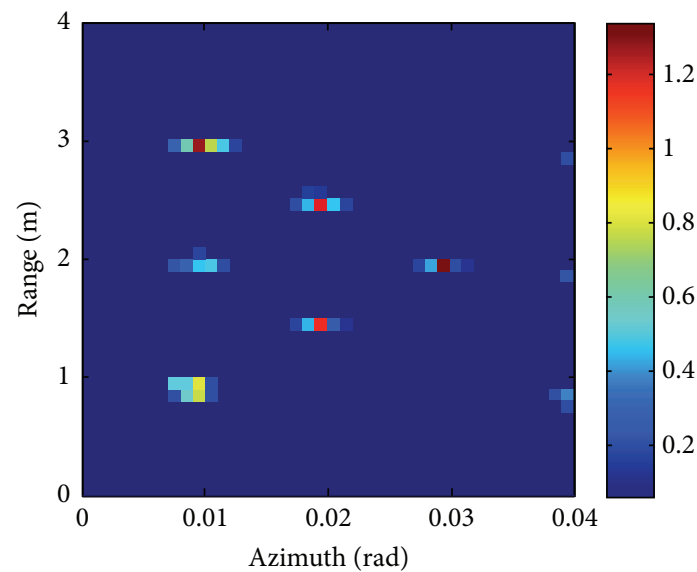

(c)

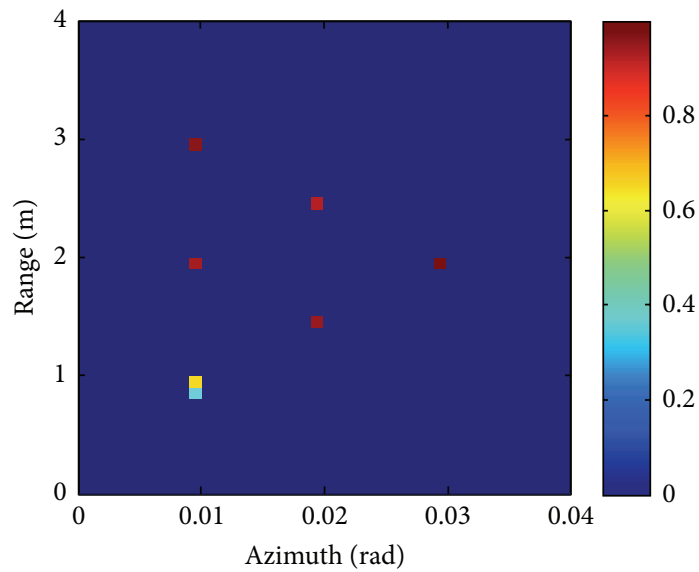

(e)

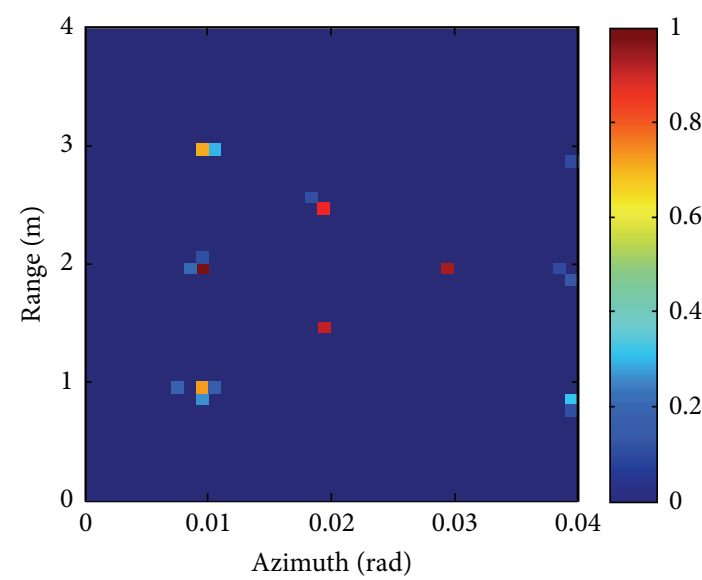

(b)

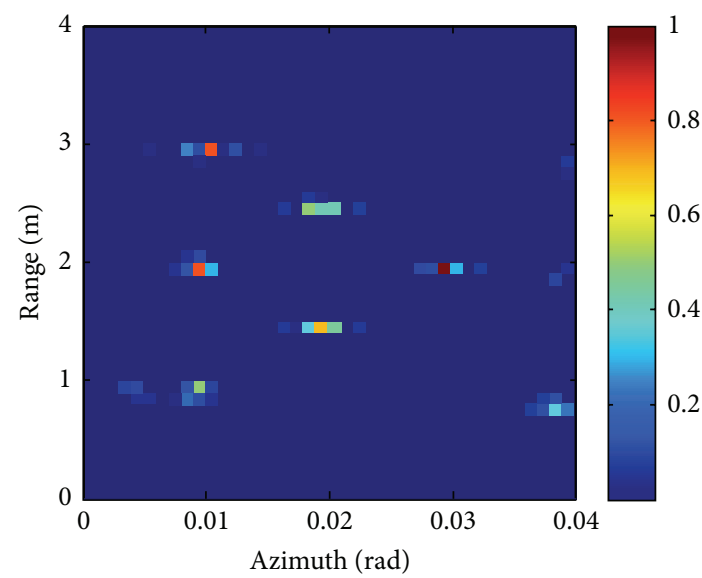

(d)

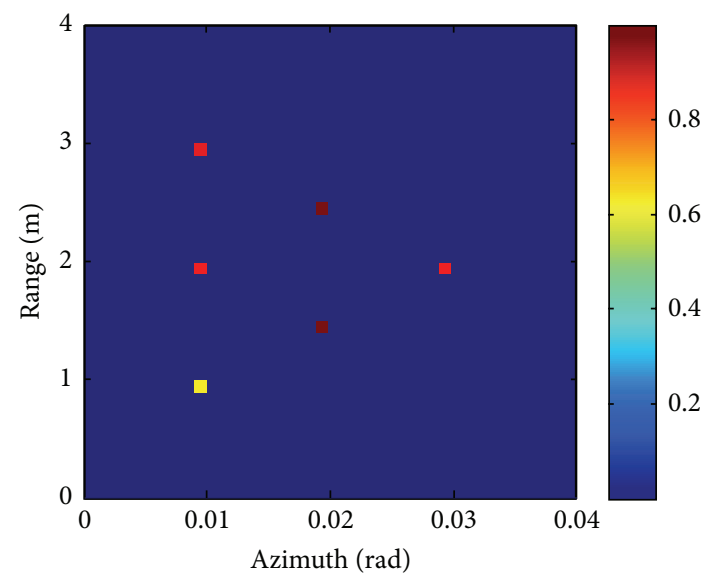

(f)

FIGURE 6: Off-grid RCI results: (a) original image; (b) BLOOMP; (c) JCP; (d) S-TLS; (e) BSBL; (f) OG-VSBL.

due to high order terms of Taylor expansion, which also render a smaller RIE consequently. Besides, the RIE obtained by OG-VSBL is much lower than that obtained by other algorithms, which shows the performance advantage of OGVSBL. Consequently, the grid size should be chosen carefully when the imaging scene is discretized.

\section{Conclusion}

In this paper, we investigate the off-grid RCI based on VSBL framework. For sparse-based RCI, the off-grid effect would lead to the imaging performance deterioration significantly. To improve the imaging performance, we approximate 


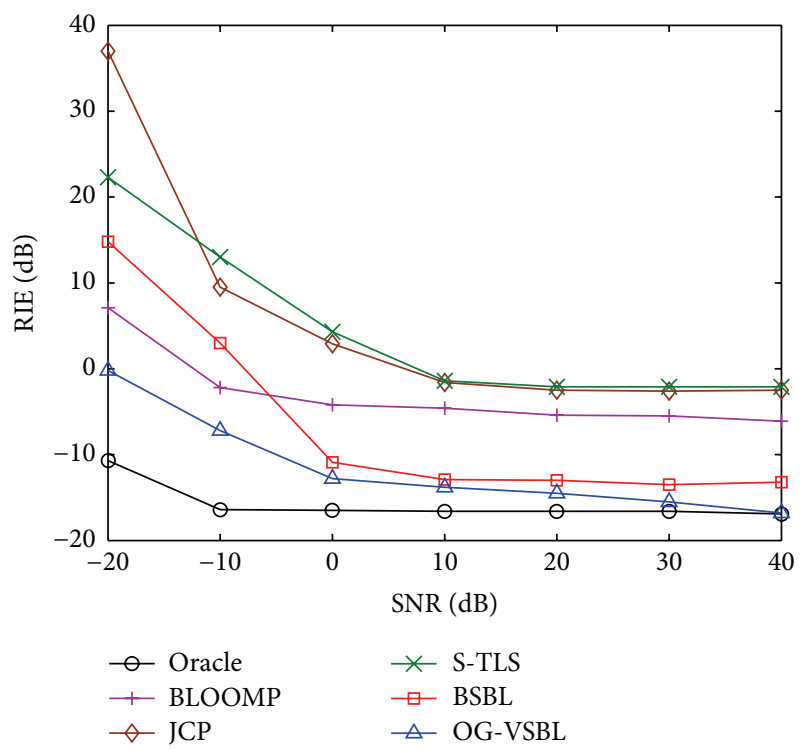

FIGURE 7: RIE versus SNR in the presence of off-grid scatterers.

the off-grid RCI model linearly using the Taylor expansion, and then an accurate model for sparse approximation is established. Based on this model, target reconstruction is recast as a joint sparse recovery problem that recovers three groups of coefficients over three known dictionaries subject to the constraint that the three groups share the same support. Such a problem can be readily manipulated from a Bayesian perspective; thus we developed an algorithm based on VSBL framework to solve the problem. Numerical experiments illustrate that this algorithm can exhibit outstanding performance, which shows the potential for the algorithm to be applied in practical radar system.

Although grid pruning is applied, the proposed algorithm still suffers from high computational complexity especially when the dictionary has large numbers of atoms in the case of 3D RCI or dense discretized grids. Then developing fast versions of OG-VSBL are of great interest, and the fast VSBL scheme in [15] could be a candidate. Meanwhile, OG-VSBL is sensitive to the RCI model parameters; then the effects of model parameters would be investigated. Besides, the algorithm achieves grid matching, while the exact locations of scatterers are ignored. Thus OGE estimation should be executed to reconstruct the target exactly, which would make the imaging results more outstanding. These open issues are beyond the scope of this paper and will be investigated deeply in the near future.

\section{Competing Interests}

The authors declare that they have no competing interests.

\section{Acknowledgments}

This work was supported by the National Natural Science Foundation of China (nos. 61302149 and 61302142) and
Research Fund for the Doctoral Program of Higher Education of China (20124307110013).

\section{References}

[1] D. Li, X. Li, Y. Cheng, Y. Qin, and H. Wang, "Radar coincidence imaging: an instantaneous imaging technique with stochastic signals," IEEE Transactions on Geoscience and Remote Sensing, vol. 52, no. 4, pp. 2261-2277, 2014.

[2] D. Li, X. Li, Y. Cheng, Y. Qin, and H. Wang, "Radar coincidence imaging in the presence of target-motion-induced error," Journal of Electronic Imaging, vol. 23, no. 2, Article ID 023014, 2014.

[3] D. Li, X. Li, Y. Cheng, Y. Qin, and H. Wang, "Radar coincidence imaging under grid mismatch," ISRN Signal Processing, vol. 2014, Article ID 987803, 8 pages, 2014.

[4] D. L. Donoho, "Compressed sensing," IEEE Transactions on Information Theory, vol. 52, no. 4, pp. 1289-1306, 2006.

[5] S. Qaisar, R. M. Bilal, W. Iqbal, M. Naureen, and S. Lee, "Compressive sensing: from theory to applications, a survey," Journal of Communications and Networks, vol. 15, no. 5, pp. 443456, 2013.

[6] X. He, C. Liuc, B. Liu, and D. Wang, "Sparse frequency diverse MIMO radar imaging for off-grid target based on adaptive iterative MAP," Remote Sensing, vol. 5, no. 2, pp. 631-647, 2013.

[7] L. Hu, J.-X. Zhou, Z.-G. Shi, and Q. Fu, "An EM-based approach for compressed sensing using dynamic dictionaries," Journal of Electronics \& Information Technology, vol. 34, no. 11, pp. 25542560, 2012.

[8] Y. Chi, L. L. Scharf, A. Pezeshki, and A. R. Calderbank, "Sensitivity to basis mismatch in compressed sensing," IEEE Transactions on Signal Processing, vol. 59, no. 5, pp. 2182-2195, 2011.

[9] X. Han, H. Zhang, and G. Li, "Fast algorithms for sparse recovery with perturbed dictionary," http://arxiv.org/abs/1111.6237.

[10] I. Kyriakides, R. Pribic, H. Sar, and N. At, "GRID matching in Monte Carlo Bayesian compressive sensing," in Proceedings of the 16th International Conference of Information Fusion (FUSION '13), pp. 2103-2109, Istanbul, Turkey, July 2013.

[11] M. A. C. Tuncer and A. C. Gurbuz, "Analysis of unknown velocity and target off the grid problems in compressive sensing based subsurface imaging," in Proceedings of the 36th IEEE International Conference on Acoustics, Speech, and Signal Processing (ICASSP '11), pp. 2880-2883, Prague, Czech Republic, May 2011.

[12] T. L. Hansen, M. A. Badiu, B. H. Fleury, and B. D. Rao, "A sparse Bayesian learning algorithm with dictionary parameter estimation," in Proceedings of the IEEE 8th Sensor Array and Multichannel Signal Processing Workshop (SAM '14), pp. 385388, A Coruña, Spain, June 2014.

[13] A. Fannjiang and H.-C. Tseng, "Compressive radar with off-grid targets: a perturbation approach," Inverse Problems, vol. 29, no. 5, Article ID 054008, 2013.

[14] A. C. Gurbuz, O. Teke, and O. Arikan, "Sparse groundpenetrating radar imaging method for off-the-grid target problem," Journal of Electronic Imaging, vol. 22, no. 2, Article ID 12317SS, 2013.

[15] G. Tang, B. N. Bhaskar, P. Shah, and B. Recht, "Compressed sensing off the grid," IEEE Transactions on Information Theory, vol. 59, no. 11, pp. 7465-7490, 2013.

[16] C. Ekanadham, D. Tranchina, and E. P. Simoncelli, "Recovery of sparse translation-invariant signals with continuous basis 
pursuit," IEEE Transactions on Signal Processing, vol. 59, no. 10, pp. 4735-4744, 2011.

[17] A. Fannjiang and W. Liao, "Coherence-pattern-guided compressive sensing with unresolved grids," SIAM Journal on Imaging Sciences, vol. 5, no. 1, pp. 179-202, 2012.

[18] H. Zhu, G. B. Giannakis, and G. Leus, "Weighted and structured sparse total least-squares for perturbed compressive sampling," in Proceedings of the 36th IEEE International Conference on Acoustics, Speech, and Signal Processing (ICASSP '11), pp. 37923795, Prague, Czech Republic, May 2011.

[19] Z. Tan, P. Yang, and A. Nehorai, "Joint sparse recovery method for compressed sensing with structured dictionary mismatches," IEEE Transactions on Signal Processing, vol. 62, no. 19, pp. 4997-5008, 2014.

[20] Z. Yang, L. Xie, and C. Zhang, "Off-grid direction of arrival estimation using sparse Bayesian inference," IEEE Transactions on Signal Processing, vol. 61, no. 1, pp. 38-43, 2013.

[21] X. Zhou, H. Wang, Y. Cheng, Y. Qin, and X. Xu, "Off-grid radar coincidence imaging based on block sparse Bayesian learning," in Proceedings of the IEEE Workshop on Signal Processing Systems (SiPS '15), pp. 1-5, Hangzhou, China, October 2015.

[22] L. Hu, Z. Shi, J. Zhou, and Q. Fu, "Compressive high-rangeresolution radar imaging using dynamic dictionaries," IET Radar, Sonar \& Navigation, vol. 7, no. 5, pp. 497-507, 2013.

[23] L. Hu, J. Zhou, Z. Shi, and Q. Fu, "A fast and accurate reconstruction algorithm for compressed sensing of complex sinusoids," IEEE Transactions on Signal Processing, vol. 61, no. 22, pp. 5744-5754, 2013.

[24] D. G. Tzikas, A. C. Likas, and N. P. Galatsanos, “The variational approximation for Bayesian inference: life after the EM algorithm," IEEE Signal Processing Magazine, vol. 25, no. 6, pp. 131146, 2008.

[25] C. M. Bishop and M. E. Tipping, "Variational relevance vector machines," in Proceedings of the 16th Conference on Uncertainty in Artificial Intelligence (UAI '00), pp. 46-53, San Francisco, Calif, USA, 2000.

[26] T. Buchgraber and D. Shutin, "Distributed variational sparse Bayesian learning for sensor networks," in Proceedings of the 22nd IEEE International Workshop on Machine Learning for Signal Processing (MLSP '12), pp. 1-6, Santander, Spain, September 2012.

[27] M. E. Tipping, "Sparse Bayesian learning and the relevance vector machine," Journal of Machine Learning Research, vol. 1, no. 3, pp. 211-244, 2001.

[28] C.-Y. Chen and P. P. Vaidyanathan, "MIMO radar ambiguity properties and optimization using frequency-hopping waveforms," IEEE Transactions on Signal Processing, vol. 56, no. 12, pp. 5926-5936, 2008.

[29] V. U. Reddy, S. Badrinath, and A. Srinivas, "Low-complexity design of frequency-hopping codes for MIMO radar for arbitrary doppler," EURASIP Journal on Advances in Signal Processing, vol. 2010, Article ID 319065, 14 pages, 2010.

[30] S. Kwon, J. Wang, and B. Shim, "Multipath matching pursuit," IEEE Transactions on Information Theory, vol. 60, no. 5, pp. 2986-3001, 2014. 


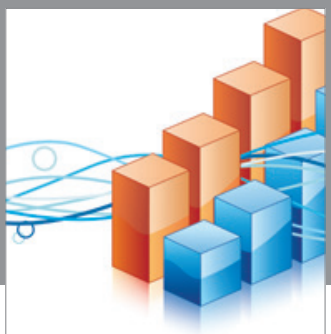

Advances in

Operations Research

vatem alat4

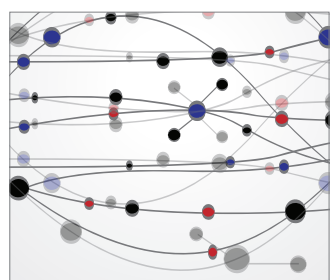

\section{The Scientific} World Journal
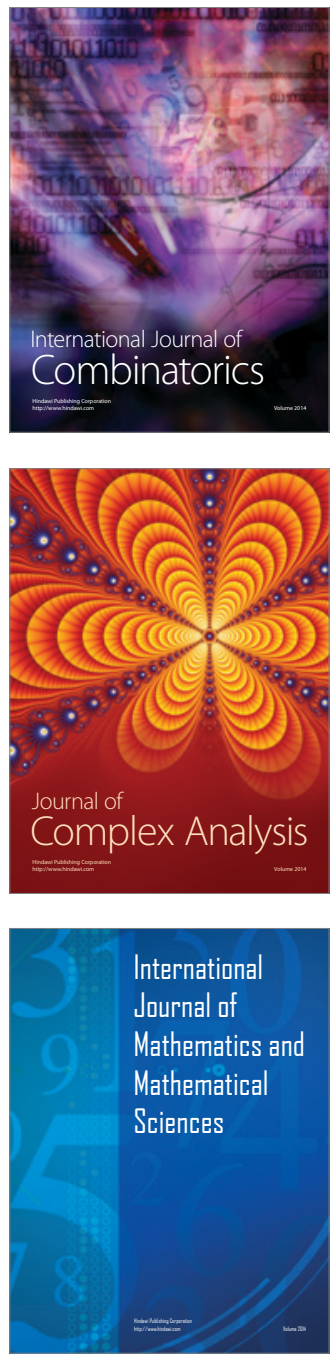
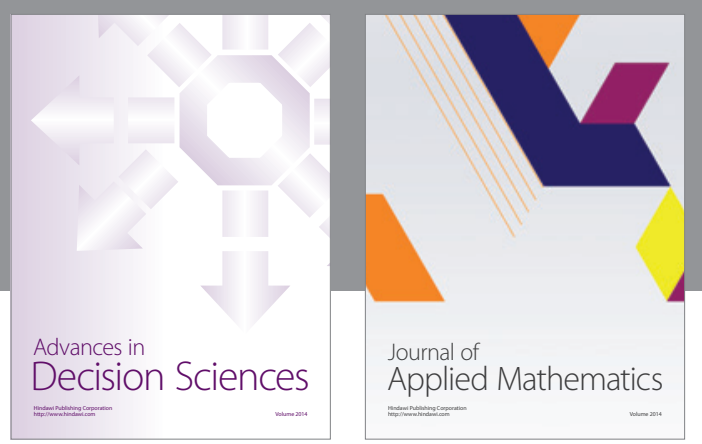

Algebra

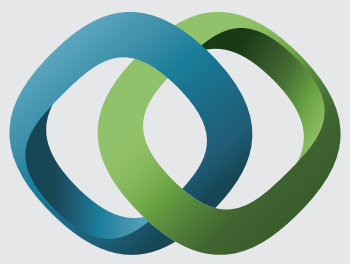

\section{Hindawi}

Submit your manuscripts at

http://www.hindawi.com
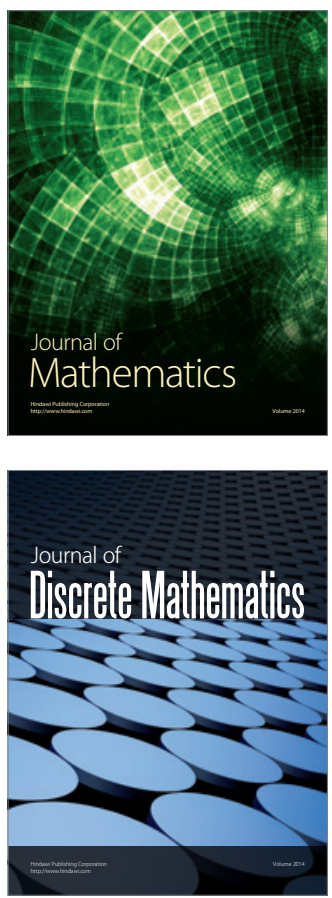

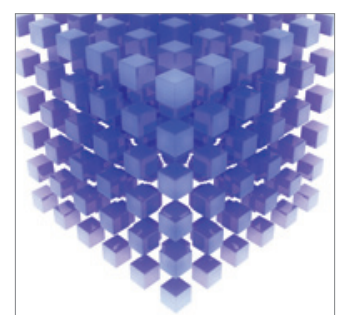

Mathematical Problems in Engineering
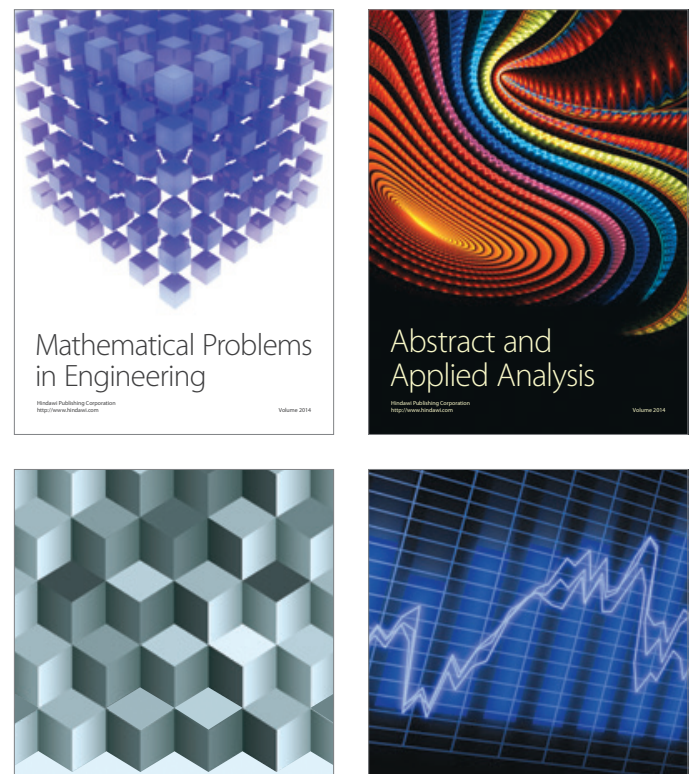

Journal of

Function Spaces

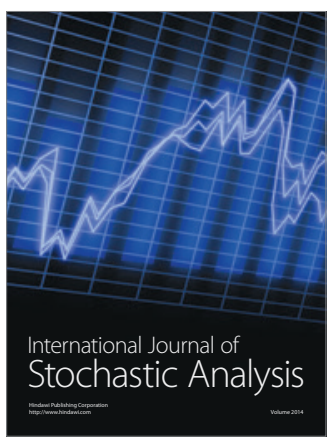


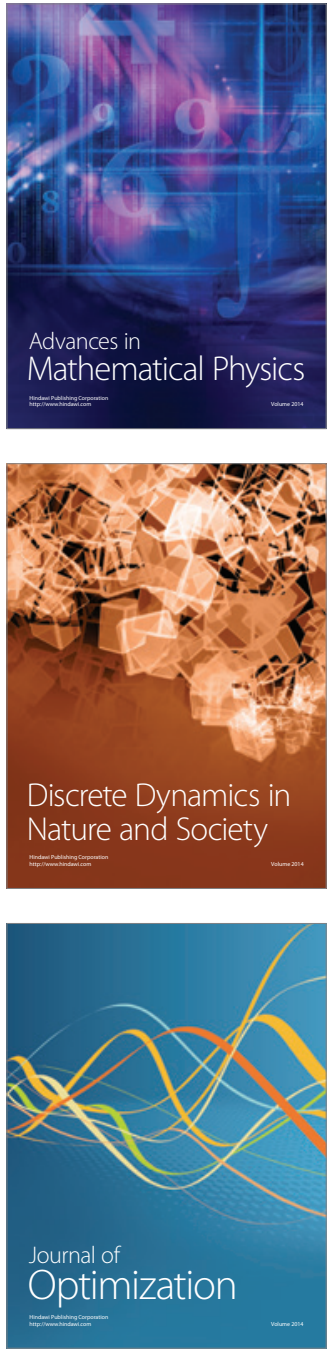